\title{
RADIATION ABSORPTION AND CHEMICAL REACTION EFFECTS ON RIVLIN-ERICKSEN FLOW PAST A VERTICAL MOVING POROUS PLATE
}

\author{
C.S. SRAVANTHI \\ Department of Mathematics, The Maharaja Sayajirao University of Baroda \\ Vadodara-390002, Gujarat, INDIA \\ E-mail: srinivasan_sravanthi@yahoo.com \\ R.S.R. GORLA* \\ Department of Mechanical Engineering, Cleveland State University \\ Cleveland, OHIO, 44115 USA \\ E-mail: r.gorla@yahoo.com
}

\begin{abstract}
An analysis has been carried out to study the combined effects of radiation absorption and chemical reaction on an incompressible, electrically conducting and radiating flow of a Rivlin-Ericksen fluid along a semi-infinite vertical permeable moving plate in the presence of a transverse applied magnetic field. It is assumed that the suction velocity, the temperature and the concentration at the wall are exponentially varying with time. The dimensionless governing equations for this investigation are solved analytically using two-term harmonic and non-harmonic functions. A comparison is made with the available results in the literature for a special case and our results are in very good agreement with the known results. A parametric study of the physical parameters is made and results are presented through graphs and tables. The results indicate that the fluid velocity and temperature could be controlled by varying the radiation absorption.
\end{abstract}

Key words: Rivlin-Ericksen fluid, radiation absorption, chemical reaction, variable temperature, concentration and suction.

\section{Introduction}

Fluids with the elasticity property of solids as well as viscous property of liquids are called viscoelastic fluids. These fluid flows have numerous and wide-ranging applications in various fields, e.g., petrochemical, biomedical and environmental engineering including polypropylene coalescence sintering, dynamically -loaded journal bearings, blood flow and geological flows [1-4].

Visco-elastic fluid flow through porous media has applications in the field of petroleum engineering in the field of chemical engineering for the purification and filtration processes, in studies on drug permeation through human skin, and in the field of hydrology for the study of migration of underground water. Many researchers [5-10] have paid their attention to-the study of visco-elastic fluid flow through a porous medium.

There are many visco-elastic fluids that cannot be characterised by Maxwell's or Oldroyd's constitutive relations. One such class of visco-elastic fluids is the Rivlin-Ericksen fluid. Rivlin and Ericksen [11] proposed a theoretical model for such elastic-viscous fluids. The Rivlin-Ericksen visco-elastic fluid has applications in geophysical fluid dynamics, chemical technology and industry. Srivastava and Singh [12] studied the unsteady flow of a dusty elastico-viscous Rivlin-Ericksen fluid through channels of different cross-sections in the presence of a time-dependent pressure gradient. Garg et al. [13] studied the rectilinear

\footnotetext{
* To whom correspondence should be addressed
} 
oscillations of a sphere along its diameter in a conducting dusty Rivlin-Ericksen fluid in the presence of uniform magnetic field. Sharma and Kango [14] investigated the thermal convection in a Rivlin-Ericksen elastico-viscous fluid in a porous medium in the presence of a uniform magnetic field. Many articles on the Rivlin-Ericksen fluid flow through a porous medium are available in the literature [15-19].

Kim [20] investigated an unsteady MHD convective heat transfer past a semi-infinite vertical porous moving plate with variable suction. In this study, the fluid considered was Newtonian. Ravikumar et al. [21] extended the study of Kim [20], by considering the Rivlin-Ericksen fluid for the case of a semi-infinite moving porous plate in the presence of pressure gradient and constant velocity in the flow direction when the magnetic field is imposed transversely to the plate.

No attempt has been made so far to analyze the boundary layer flow of unsteady MHD mixed convective heat and mass transfer flow of a Rivlin-Ericken fluid along a vertical permeable moving porous plate with chemical reaction and radiation absorption with variable suction, temperature and concentration. The present work aims to fill the gap in the existing literature. The governing boundary layer equations are solved analytically using two-term harmonic and non- harmonic functions. The effects of different emerging parameters are demonstrated through graphical representations and discussed at length.

\section{Formulation of problem}

Consider an unsteady two-dimensional laminar flow of an incompressible electrically conducting Rivlin-Ericksen fluid past a permeable semi infinite vertical moving plate embedded in a porous medium. The $x^{*}$ - axis is taken along the vertical plate and the $y^{*}$-axis is normal to the plate (see Fig.1). A uniform magnetic field of strength $B_{0}$ is applied in the $y^{*}$-direction. The magnetic Reynolds number is assumed to be very small so the induced magnetic field is neglected. In addition, as there is no applied electric field Joule heating and Hall effects are also neglected. In the present analysis, thermal radiation, radiation absorption and first order chemical reaction effects are taken into account. Due to the assumption that the plate is semi-infinite, the flow variables are functions of normal distance $y^{*}$ and $t^{*}$ only. Under the above assumptions, the governing equations are given by [21], [22]

$$
\begin{aligned}
& \frac{\partial v^{*}}{\partial y^{*}}=0 \\
& \frac{\partial u^{*}}{\partial t^{*}}+v^{*} \frac{\partial u^{*}}{\partial y^{*}}=-\frac{1}{\rho} \frac{\partial p^{*}}{\partial x^{*}}+v \frac{\partial^{2} u^{*}}{\partial y^{* 2}}+g \beta_{T}\left(T^{*}-T_{\infty}^{*}\right)+ \\
& +g \beta_{C}\left(C^{*}-C_{\infty}^{*}\right)-v \frac{u^{*}}{k^{*}}-\frac{\sigma B_{0}^{2} u^{*}}{\rho}-\beta_{I}\left(\frac{\partial^{3} u^{*}}{\partial t^{*} \partial y^{* 2}}+v^{*} \frac{\partial^{3} u^{*}}{\partial y^{* 3}}\right), \\
& \frac{\partial T^{*}}{\partial t^{*}}+v^{*} \frac{\partial T^{*}}{\partial y^{*}}=\alpha \frac{\partial^{2} T^{*}}{\partial y^{* 2}}-\frac{1}{\rho c_{p}} \frac{\partial q_{r}^{*}}{\partial y^{*}}+Q_{c}^{*}\left(C^{*}-C_{\infty}^{*}\right) \\
& \frac{\partial C^{*}}{\partial t^{*}}+v^{*} \frac{\partial C^{*}}{\partial y^{*}}=D \frac{\partial^{2} T^{*}}{\partial y^{* 2}}-K_{l}\left(C^{*}-C_{\infty}^{*}\right)
\end{aligned}
$$




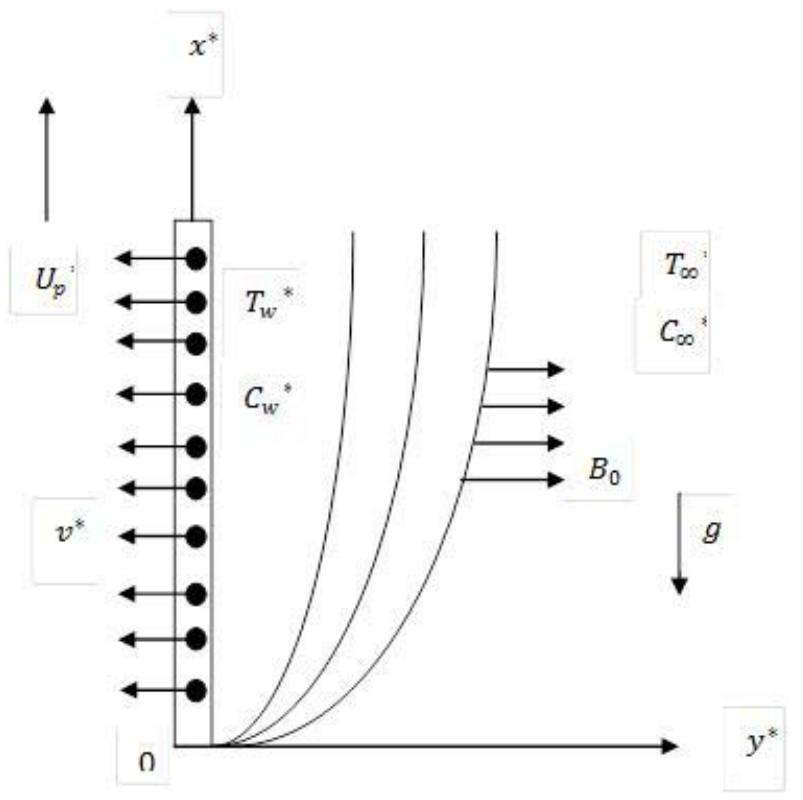

Fig.1. Schematic diagram of the physical model.

It is assumed that the permeable plate moves with a constant velocity in the direction of fluid flow and the free stream velocity follows the exponentially increasing small perturbation law. In addition, it is also assumed that the temperature and the concentration at the wall as well as the suction velocity are exponentially varying with time. With these assumptions the boundary conditions for the velocity, temperature and concentration fields are [21]

$$
\begin{aligned}
& u^{*}=U_{p}^{*}, \quad T^{*}=T_{w}^{*}+\varepsilon\left(T_{\omega}^{*}-T_{\infty}^{*}\right) e^{n^{* *} t^{*}}, \quad C^{*}=C_{w}^{*}+\varepsilon\left(C_{\omega}^{*}-C_{\infty}^{*}\right) e^{n^{* *}} \text { at } y^{*}=0, \\
& u^{*}=U_{\infty}^{*}=U_{0}\left(1+\varepsilon e^{n^{*} t^{*}}\right), \quad T^{*} \rightarrow T_{\infty}^{*}, \quad C^{*} \rightarrow C_{\infty}^{*} \quad \text { as } \quad y^{*} \rightarrow \infty
\end{aligned}
$$

It is clear from Eq.(2.1) that the suction velocity at the plate is either a constant or a function of time. Hence, the suction velocity normal to the plate is assumed in the form

$$
v^{*}=-v_{0}\left(1+\varepsilon A e^{n^{* *} t^{*}}\right)
$$

where $v_{0}$ is the scale of suction velocity which is a non-zero positive constant. The negative sign indicates that the suction is towards the plate.

Outside the boundary layer, Eq.(2.2) gives

$$
-\frac{1}{\rho} \frac{\partial p^{*}}{\partial x^{*}}=\frac{d U_{\infty}^{*}}{d t^{*}}+\frac{\mathcal{V}}{K^{*}} U_{\infty}^{*}+\frac{\sigma B_{0}^{2} U_{\infty}^{*}}{\rho} .
$$

For the case of an optically thin gray gas, the radiative heat flux in energy Eq.(2.5) in the spirit of Vincenti and Krugger [23] is expressed as 


$$
\frac{\partial q_{r}^{*}}{\partial y^{*}}=-4 a^{*} \sigma^{*}\left(T_{\infty}^{* 4}-T^{* 4}\right) .
$$

It is assumed that the temperature differences within the flow are sufficiently small such that $T^{* 4}$ may be expressed as a linear function of the temperature $T^{*}$.This is accomplished by expanding $T^{* 4}$ in Taylor series about $T_{\infty}^{*}$ and neglecting higher order terms, thus

$$
T^{* 4} \cong 4 T_{\infty}^{* 3} T^{*}-3 T_{\infty}^{*}{ }^{4}
$$

By substituting Eq.(2.9) in Eq.(2.8) we obtain

$$
\frac{\partial q_{r}{ }^{*}}{\partial y^{*}}=16 a^{*} \sigma^{*} T_{\infty}^{* 3}\left(T^{*}-T_{\infty}^{*}\right) \text {. }
$$

In order to write the governing equations and boundary conditions in dimensionless form, the following non-dimensional quantities are introduced.

$$
\begin{aligned}
& U=\frac{u^{*}}{U_{0}}, \quad \eta=\frac{V_{0} y^{*}}{v}, \quad U_{\infty}=\frac{U_{\infty}^{*}}{U_{0}}, \quad U_{p}=\frac{U_{p}^{*}}{U_{0}}, \quad t=\frac{t^{*} V_{0}^{2}}{\mathcal{V}}, \quad \theta=\frac{T^{*}-T_{\infty}^{*}}{T_{w}^{*}-T_{\infty}^{*}}, \\
& R=\frac{16 \sigma^{*} a^{*} T_{\infty}^{*} \mathcal{V}}{\rho c_{p} V_{0}^{2}}, \quad R m=\frac{\beta_{1} V_{0}^{2}}{v^{2}}, \quad C=\frac{C^{*}-C_{\infty}^{*}}{C_{w}^{*}-C_{\infty}^{*}}, \quad K=\frac{k^{*} V_{0}^{2}}{v^{2}}, n=\frac{n^{*} v}{V_{0}^{2}}, \\
& \operatorname{Pr}=\frac{v}{\alpha}=\frac{v \rho c_{p}}{k}, \quad Q c=\frac{Q_{C}^{*} v\left(C_{w}^{*}-C_{\infty}^{*}\right)}{V_{0}^{2}\left(T_{w}^{*}-T_{\infty}^{*}\right)}, \quad k r=\frac{K_{1} v}{V_{0}^{2}}, \\
& M=\frac{\sigma B_{0}^{2} v}{\rho V_{0}^{2}}, \quad \mathrm{Gr}=\frac{v g \beta_{T}\left(T_{w}^{*}-T_{\infty}^{*}\right)}{U_{0} V_{0}^{2}}, \quad \mathrm{Gc}=\frac{v g \beta_{C}\left(C_{w}^{*}-C_{\infty}^{*}\right)}{U_{0} V_{0}^{2}}, \quad \mathrm{Sc}=\frac{v}{D} .
\end{aligned}
$$

In view of Eqs (2.6)-(2.11), Eqs (2.2)-(2.4) reduce to the following dimensionless form.

$$
\begin{aligned}
& \frac{\partial U}{\partial t}-\left(1+\varepsilon A e^{n t}\right) \frac{\partial U}{\partial \eta}=\frac{d U_{\infty}}{d t}+N\left(U_{\infty}-U\right)+\frac{\partial^{2} U}{\partial \eta^{2}}+ \\
& +\mathrm{Gr} \theta+\operatorname{Gc} C-R m\left(\frac{\partial^{3} U}{\partial t \partial \eta^{2}}-\left(1+\varepsilon A e^{n t}\right) \frac{\partial^{3} U}{\partial \eta^{3}}\right), \\
& \frac{\partial \theta}{\partial t}-\left(1+\varepsilon A e^{n t}\right) \frac{\partial \theta}{\partial \eta}=\frac{1}{\operatorname{Pr}} \frac{\partial^{2} \theta}{\partial \eta^{2}}-R \theta+Q c C,
\end{aligned}
$$




$$
\frac{\partial C}{\partial t}-\left(1+\varepsilon A e^{n t}\right) \frac{\partial C}{\partial \eta}=\frac{1}{\operatorname{Sc}} \frac{\partial^{2} C}{\partial \eta^{2}}-K r C
$$

where

$$
N=M+\frac{1}{K}
$$

The corresponding boundary conditions are

$$
\begin{aligned}
& U=U_{p}, \quad T=1+\varepsilon e^{n t}, \quad C=1+\varepsilon e^{n t} \quad \text { at } \quad \eta=0, \\
& U=U_{\infty}=1+\varepsilon e^{n t}, \quad T \rightarrow 0, \quad C \rightarrow 0 \quad \text { as } \quad \eta \rightarrow \infty .
\end{aligned}
$$

\section{Solution of the problem}

Equations (2.12)-(2.14) are coupled, non-linear partial differential equations and these cannot be solved in closed-form. However, these equations can be reduced to a set of ordinary differential equations, which can be solved analytically. This can be done by representing the velocity, temperature and concentration of the fluid in the neighbourhood of the plate as

$$
\begin{aligned}
& U=f_{0}(\eta)+\varepsilon e^{n t} f_{l}(\eta)+O\left(\varepsilon^{2}\right)+\ldots, \\
& \theta=g_{0}(\eta)+\varepsilon e^{n t} g_{l}(\eta)+O\left(\varepsilon^{2}\right)+\ldots, \\
& C=h_{0}(\eta)+\varepsilon e^{n t} h_{l}(\eta)+O\left(\varepsilon^{2}\right)+\ldots
\end{aligned}
$$

Substituting Eq.(3.1) in Eqs (2.12)-(2.14) and equating harmonic and non-harmonic terms, and neglecting the higher order terms of $O\left(\varepsilon^{2}\right)$, we obtain

$$
\begin{aligned}
& R m f_{0}^{\prime \prime \prime}+f_{0}^{\prime \prime}+f_{0}^{\prime}-N f_{0}=-\operatorname{Gr} g_{0}-G c h_{0}-N, \\
& R m f_{1}^{\prime \prime \prime}+\left(1-n R_{m}\right) f_{1}^{\prime \prime}+f_{1}^{\prime}-(N+n) f_{1}=-n-N-A f_{0}^{\prime}-\mathrm{Grg}_{1}-G c h_{1}-R m A f_{0}^{\prime \prime \prime}, \\
& g_{0}^{\prime \prime}+\operatorname{Pr} g_{0}^{\prime}-R \operatorname{Pr} g_{0}=-Q c \operatorname{Pr} h_{0}, \\
& g_{1}^{\prime \prime}+\operatorname{Pr} g_{1}^{\prime}-(R+n) \operatorname{Pr} g_{1}=-Q c \operatorname{Pr} h_{1}-g_{0}^{\prime} \operatorname{Pr} A, \\
& h_{0}^{\prime \prime}+\operatorname{Sc} h_{0}^{\prime}-K r \operatorname{Sc} h_{0}=0, \\
& h_{1}^{\prime \prime}+\operatorname{Sc} h_{1}^{\prime}-(K r+n) \operatorname{Sc} h_{1}=-h_{0}^{\prime} \operatorname{Sc} A
\end{aligned}
$$

where prime denotes ordinary differentiation with respect to $\eta$. The corresponding boundary conditions can be written as 


$$
\begin{aligned}
& f_{0}=U_{p}, \quad f_{1}=0, \quad g_{0}=1, \quad g_{1}=1, \quad h_{0}=1, \quad h_{1}=1 \quad \text { at } \quad \eta=0, \\
& f_{0}=1, \quad f_{1}=1, \quad g_{0} \rightarrow 1, \quad g_{1} \rightarrow 1, \quad h_{0} \rightarrow 1, \quad h_{1} \rightarrow 1 \quad \text { as } \quad \eta \rightarrow \infty .
\end{aligned}
$$

The solutions of Eqs (3.4) - (3.7) subject to the boundary conditions (3.8) are

$$
\begin{aligned}
& g_{0}=k_{4} e^{-m_{6} \eta}+k_{3} e^{-m_{2} \eta}, \\
& g_{1}=k_{8} e^{-m_{8} \eta}+k_{5} e^{-m_{6} \eta}+k_{6} e^{-m_{4} \eta}+k_{7} e^{-m_{2} \eta}, \\
& h_{0}=e^{-m_{2} \eta}, \\
& h_{1}=k_{2} e^{-m_{4} \eta}+k_{1} e^{-m_{2} \eta} .
\end{aligned}
$$

Equations (3.2) and (3.3) are third order differential equations when $R m \neq 0$ and we have two boundary conditions, so we follow Beard and Walters [24] as

$$
\begin{aligned}
& f_{0}=f_{01}+R m f_{02}+O\left(R m^{2}\right), \\
& f_{1}=f_{11}+R m f_{12}+O\left(R m^{2}\right) .
\end{aligned}
$$

Substituting Eqs (3.13) and (3.14) into Eqs (3.2) and (3.3), equating different powers of $R m$ and neglecting $O\left(R \mathrm{~m}^{2}\right)$, we obtain

$$
\begin{aligned}
& f_{01}^{\prime \prime}+f_{01}^{\prime}-N f_{01}=-\mathrm{Gr} k_{4} e^{-m_{6} \eta}-\left(\mathrm{Gr} k_{3}+\mathrm{Gc}\right) e^{-m_{2} \eta}-N, \\
& f_{02}^{\prime \prime}+f_{02}^{\prime}-N f_{02}=-f_{01}^{\prime \prime \prime}, \\
& f_{11}^{\prime \prime}+f_{11}^{\prime}-(N+n) f_{11}=-n-N-A f_{01}^{\prime}-\mathrm{Gr} g_{1}-\mathrm{Gc} h_{1}, \\
& f_{11}^{\prime \prime \prime}+f_{12}^{\prime \prime}-n f_{11}^{\prime \prime}+f_{12}^{\prime}-(N+n) f_{12}=-A f_{02}^{\prime}-A f_{01}^{\prime \prime \prime},
\end{aligned}
$$

and the corresponding boundary conditions are

$$
\begin{aligned}
& f_{01}=U_{p}, \quad f_{02}=0, \quad f_{11}=0, \quad f_{12}=0 \quad \text { at } \quad \eta=0, \\
& f_{01}=1, \quad f_{02}=0, \quad f_{11}=1, \quad f_{12}=0 \quad \text { as } \quad \eta \rightarrow \infty .
\end{aligned}
$$

Solving Eqs (3.15) - (3.3.18) under the boundary conditions (3.19), we obtain solutions as

$$
f_{01}=k_{11} e^{-m_{10} \eta}+k_{9} e^{-m_{6} \eta}+k_{10} e^{-m_{2} \eta}+1,
$$




$$
\begin{aligned}
& f_{02}=\left(k_{15}+k_{12}\right) e^{-m_{10} \eta}+k_{13} e^{-m_{6} \eta}+k_{14} e^{-m_{2} \eta}, \\
& f_{11}=k_{21} e^{-m_{12} \eta}+k_{16} e^{-m_{10} \eta}+k_{17} e^{-m_{6} \eta}+k_{18} e^{-m_{2} \eta}+k_{19} e^{-m_{8} \eta}+k_{20} e^{-m_{4} \eta}+1, \\
& f_{12}=\left(k_{2815 m}+k_{22}\right) e^{-m_{12} \eta}+k_{23} e^{-m_{10} \eta}+k_{24} e^{-m_{6} \eta}+k_{25} e^{-m_{2} \eta}+k_{1826} e^{-m_{8} \eta}+k_{1827} e^{-m_{4} \eta} .
\end{aligned}
$$

In view of the above solutions, the velocity, temperature and concentration distributions in the boundary layer become

$$
\begin{aligned}
& C(\eta, t)=h_{0}(\eta)+\varepsilon e^{n t} h_{1}(\eta)=e^{-m_{2} \eta}+\varepsilon e^{n t}\left(k_{2} e^{-m_{4} \eta}+k_{1} e^{-m_{2} \eta}\right), \\
& \theta(\eta, t)=g_{0}(\eta)+\varepsilon e^{n t} g_{1}(\eta)=k_{4} e^{-m_{6} \eta}+k_{3} e^{-m_{2} \eta}+ \\
& +\varepsilon e^{n t}\left(k_{8} e^{-m_{8} \eta}+k_{5} e^{-m_{6} \eta}+k_{6} e^{-m_{4} \eta}+k_{7} e^{-m_{2} \eta}\right), \\
& U(\eta, t)=f_{0}(\eta)+\varepsilon e^{n t} f_{1}(\eta)=\left(f_{01}+R m f_{02}\right)+\varepsilon e^{n t}\left(f_{11}+R m f_{12}\right)= \\
& =\left(k_{11} e^{-m_{10} \eta}+k_{9} e^{-m_{6} \eta}+k_{10} e^{-m_{2} \eta}+1\right)+R m\left(\left(k_{15}+k_{12}\right) e^{-m_{10} \eta}+k_{13} e^{-m_{6} \eta}+k_{14} e^{-m_{2} \eta}\right)+ \\
& +\varepsilon e^{n t}\left(\left(k_{21} e^{-m_{12} \eta}+k_{16} e^{-m_{10} \eta}+k_{17} e^{-m_{6} \eta}+k_{18} e^{-m_{2} \eta}+k_{19} e^{-m_{8} \eta}+k_{20} e^{-m_{4} \eta}+1\right)+\right. \\
& \left.+R m\left(\left(k_{2815 m(}+k_{22}\right) e^{-m_{12} \eta}+k_{23} e^{-m_{10} \eta}+k_{24} e^{-m_{6} \eta}+k_{25} e^{-m_{2} \eta}+k_{1826} e^{-m_{8} \eta}+k_{1827} e^{-m_{4} \eta}\right)\right)
\end{aligned}
$$

where the expressions for the constants are given in the Appendix.

The physical quantities of engineering interest such as skin-friction, Nusselt number and Sherwood number for this type of boundary layer flow can be defined and determined as follows

$$
\begin{aligned}
& \tau=\left(\frac{\partial U}{\partial \eta}\right)_{\eta=0}=-\left[m_{10} k_{11}+m_{6} k_{9}+m_{2} k_{10}+R m\left(\left(k_{15}+k_{12}\right) m_{10}+k_{13} m_{6}+k_{14} m_{2}\right)\right]+ \\
& -\varepsilon e^{n t}\left[k_{21} m_{12}+k_{16} m_{10}+k_{17} m_{6}+k_{18} m_{2}+k_{19} m_{8}+k_{20} m_{4}+\right. \\
& \left.+R m\left(\left(k_{2815 m(}+k_{22}\right) m_{12}+k_{23} m_{10}+k_{24} m_{6}+k_{25} m_{2}+k_{1826} m_{8}+k_{1827} m_{4}\right)\right] \\
& \mathrm{Nu}=\left(\frac{\partial \theta}{\partial \eta}\right)_{\eta=0}=-m_{6} k_{4}-m_{2} k_{3}+\varepsilon e^{n t}\left(-m_{8} k_{8}-m_{6} k_{5}-m_{4} k_{6}-m_{2} k_{7}\right) \\
& \mathrm{Sh}=\left(\frac{\partial C}{\partial \eta}\right)_{\eta=0}=-m_{6} k_{4}-m_{2} k_{3}+\varepsilon e^{n t}\left(-m_{8} k_{8}-m_{6} k_{5}-m_{4} k_{6}-m_{2} k_{7}\right) .
\end{aligned}
$$

\section{Results and discussion}

In order to get physical insight into the problem, the effects of various parameters encountered in the equations of the problem are analyzed with the help of Figs $2-14$, while the values of some of the physical 
parameters are taken as constants such as $\mathrm{Sc}=0.62$ (water), $\varepsilon=0.2, t=1.0, k r=0.5, R=0.1, n=1, \mathrm{Pr}=0.72$ (air), $M=2.0, k=100, \mathrm{Gr}=2.0, G c=2.0, U p=1.0, A=0.5, Q c=0.5, R m=0.05$ in all the graphs and tables. All the graphs and tables therefore correspond to these values unless specifically indicated in the appropriate graph or table.

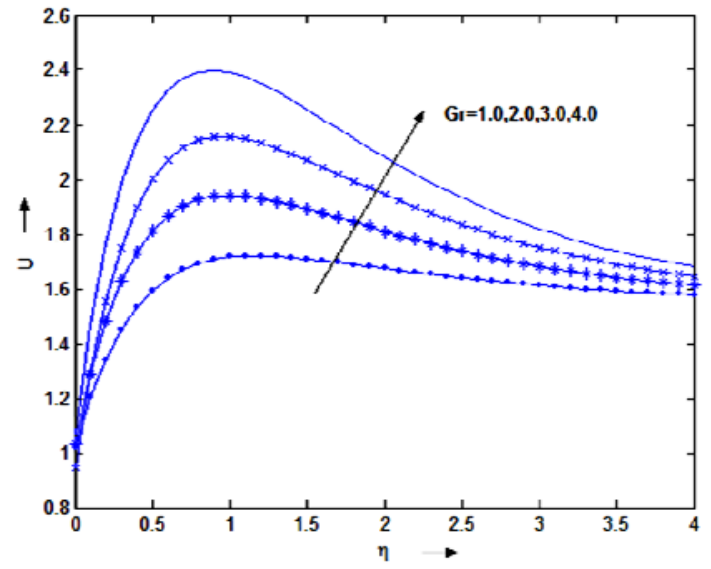

Fig.2. Effect of Gr on the velocity.

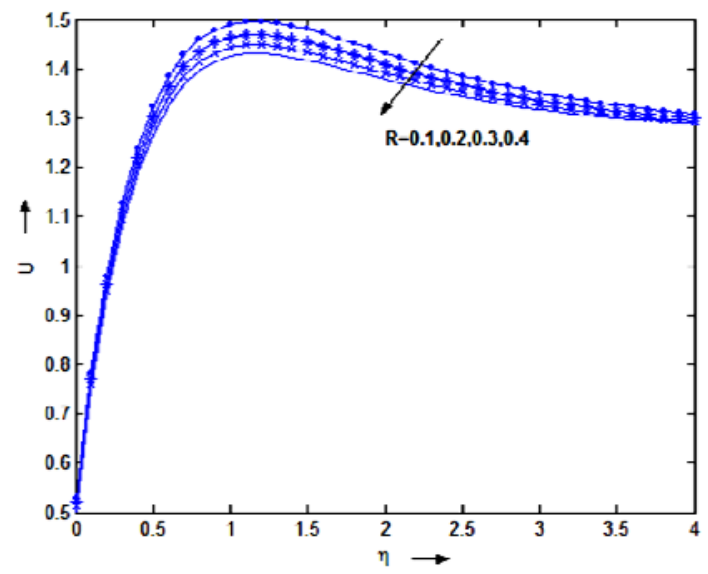

Fig..4. Effect of $R$ on the velocity.

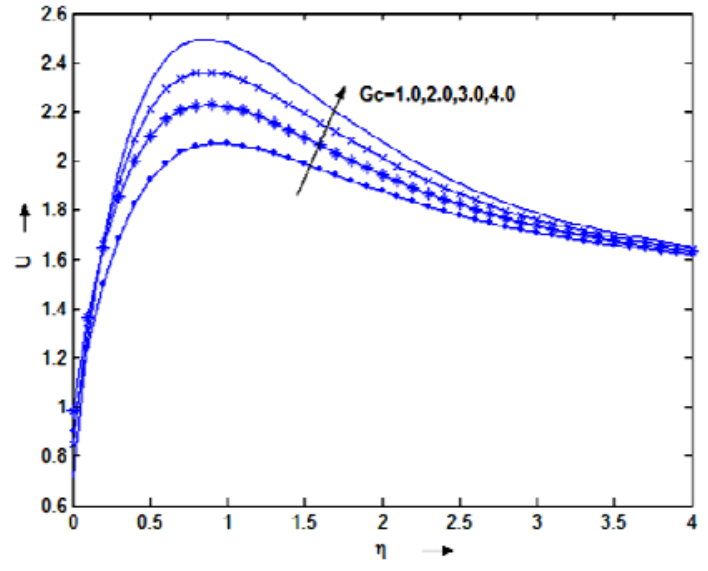

Fig.3. Effect of Gc on the velocity.

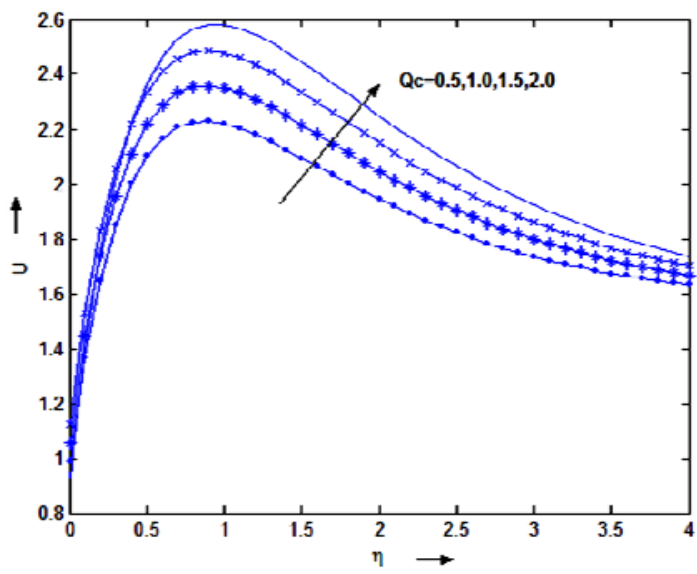

Fig.5. Effect of $Q c$ on the velocity.

Figure 2 illustrates the effect of thermal Grashof number Gr on the velocity profiles. It is noticed from the figure that an increase in Gr leads to a rise in the velocity due to the enhancement in buoyancy force. Here, the positive values of Gr correspond to cooling of the surface. The similar behavior is observed with increasing values of solutal Grashof number Gc (Fig.3) and positive values of Gc indicates that the chemical species concentration in the free stream region is less than the concentration at the boundary. The effect of the radiation parameter $R$ on the velocity field is depicted in Fig.4. It is observed that the velocity profiles decreases with an increasing values of the radiation parameter $R$. This is because of the fact that an increase in $R$ implies less interaction of radiation with the momentum boundary layer. Figure 5 depicts the effect of the radiation absorption parameter $Q c$ on the fluid velocity and it is observed that the fluid velocity increases as the $Q c$ increases. This is due to the fact when heat is absorbed, it increases the buoyancy force thereby increasing the fluid velocity. 


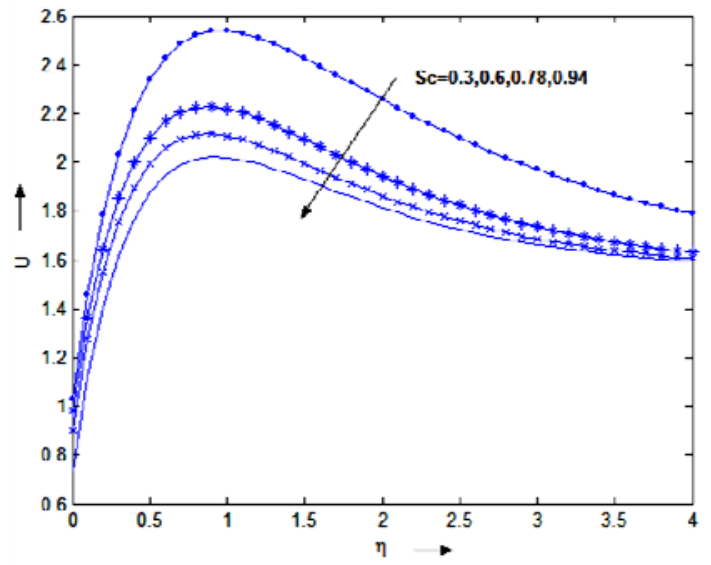

Fig.6. Effect of Sc on the velocity.

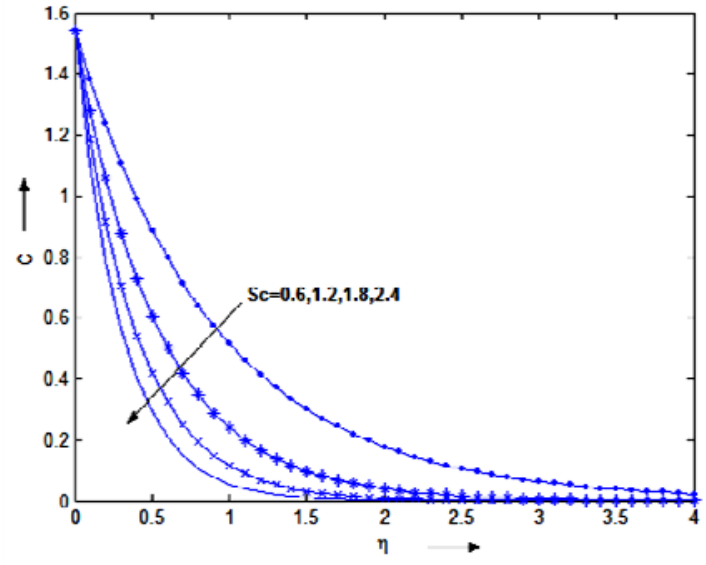

Fig.7. Effect of Sc on the concentration.

The effect of the Schmidt number Sc on the velocity and concentration are shown in Figs 6-7, respectively. The Schmidt number embodies the ratio of momentum to mass diffusivity. The Schmidt number quantifies the relative effectiveness of momentum and mass transport by diffusion in the hydrodynamic (velocity) and concentration (species) boundary layers. As the Schmidt number increases the concentration decreases. This causes the concentration buoyancy effects to decrease yielding a reduction in the fluid velocity. The reductions in the velocity and concentration profiles are accompanied by simultaneous reduction in the velocity and concentration boundary layer thicknesses.

Figure 8 illustrates the variation in velocity function for several values of the visco-elasticity parameter $R m$. It is found that an increase in $R m$ leads to a decrease in the velocity distribution across the boundary layer. This can be explained by the fact that, as the viscoelastic parameter increases, the hydromagnetic boundary layer adheres strongly to the surface, which in turn retards the flow.

Figure 9 illustrates the variation of velocity distribution across the boundary layer for several values of plate moving velocity $U_{p}$ in the direction of the fluid flow. From the figure it is interesting to note that, for different values of plate moving velocities, the velocities started at different points, but they reached to single value. That is, although we have different initial plate moving velocities, the velocity decreases to the constant value for given material parameters.

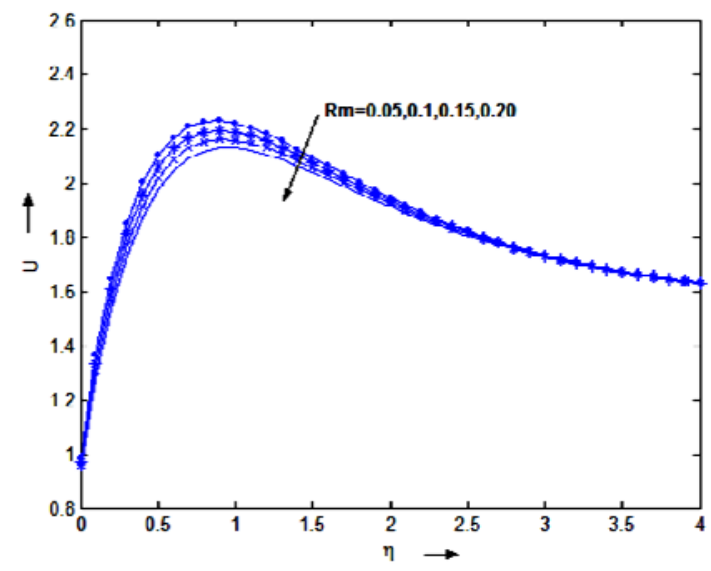

Fig.8. Effect of $R m$ on the velocity.

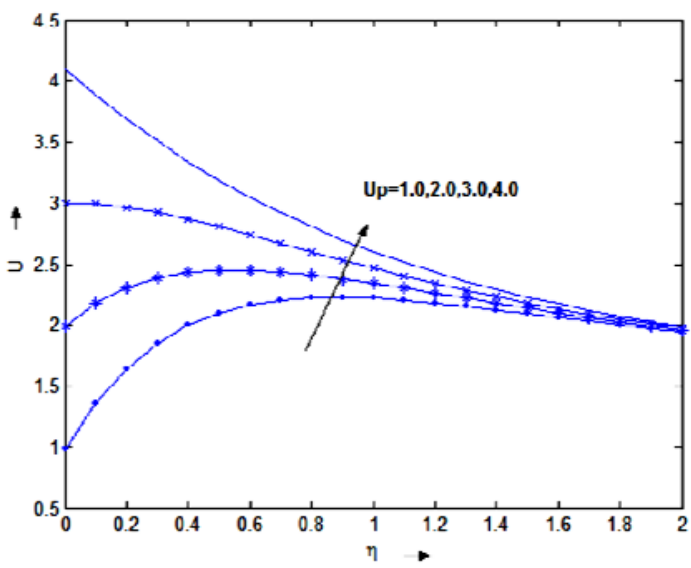

Fig.9. :Effect of $U p$ on the velocity. 
From Fig.10, it follows that an increase in the Prandtl number results in decrease in the temperature. This is, because for small values of the Pr, there will be more thermal conductivity of the fluid. Therefore, for large values of Pr, heat is able to diffuse away from the heated surface more rapidly. Hence, in the case of smaller Pr numbers the thermal boundary layer is thicker and the rate of heat transfer is reduced. Figure 11 illustrates the variation in temperature function for several values of radiation absorption parameter $Q c$. It is found that an increase in $Q c$ leads to an increase in the temperature distribution across the boundary layer. Figure 12 shows the effect of the radiation parameter $R$ on the temperature. A rise in $R$ causes a significant fall in the temperature values from the highest value at the wall across the boundary layer to the free stream. Thus, a greater value of $R$ corresponds to smaller radiation flux and the minimum temperature is observed. Radiation thereby reduces the rate of energy transport to the fluid.

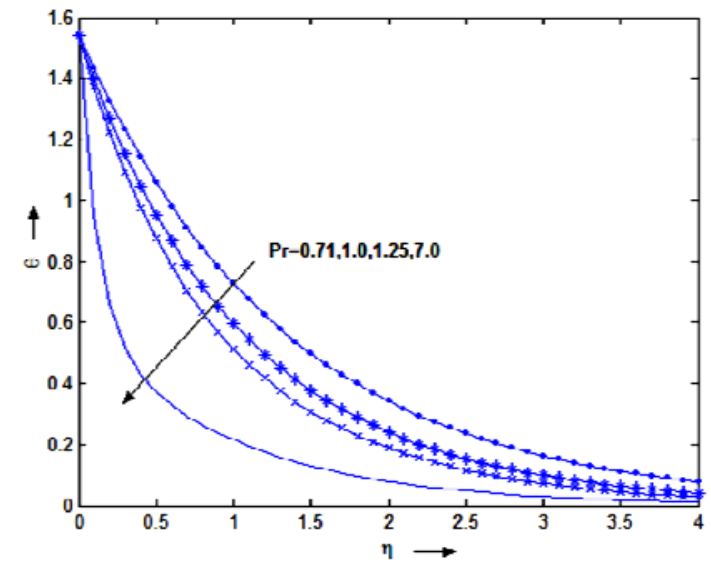

Fig.10. Effect of Pr on the temperature.

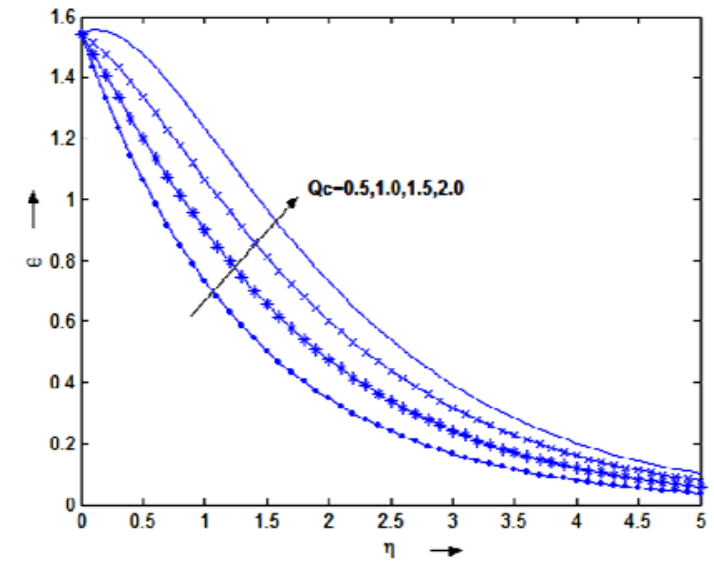

Fig.11. Effect of $Q c$ on the temperature.

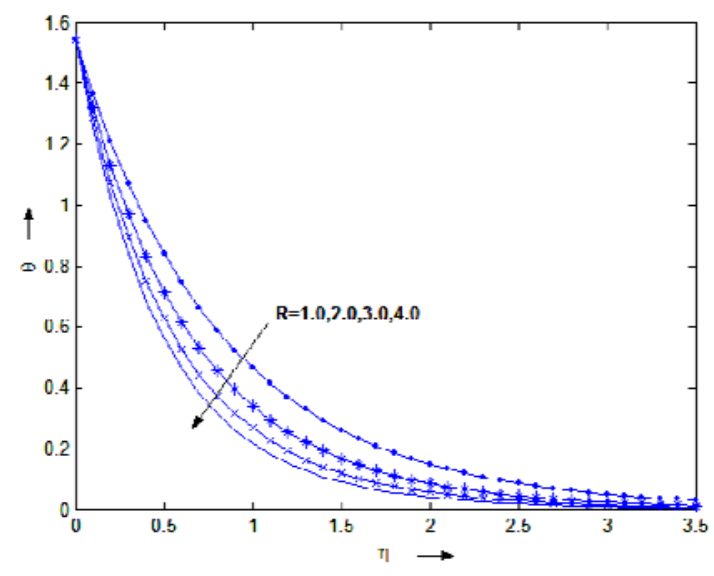

Fig.12. Effect of $R$ on the temperature.

Figure 13 shows the effect of the chemical reaction parameter $K r$ on concentration. It is noticed that species concentration and concentration boundary layer are decreasing, as the values of the chemical reaction parameter are increasing. It follows from Fig.14 that an increase in either the chemical reaction $(\mathrm{Kr})$ or Schmidt number (Sc) decreases the Sherwood number (Sh) (This is in agreement with the result of Ref. [18]). 


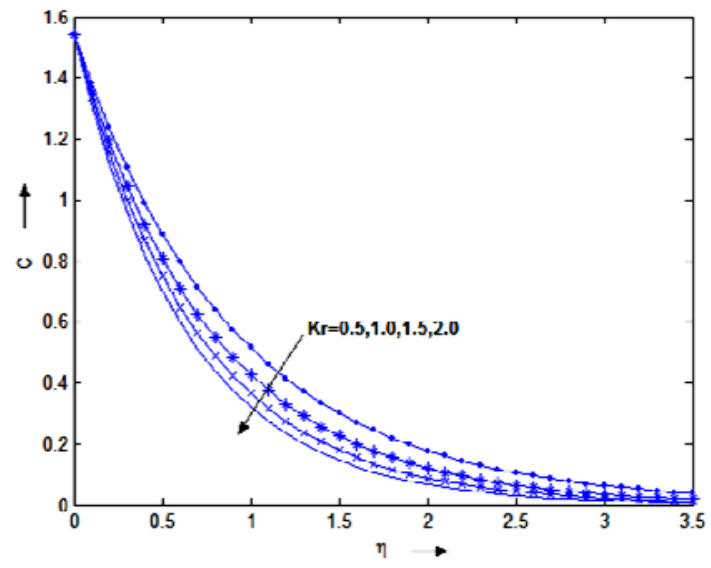

Fig.13. Effect of $K r$ on the concentration.

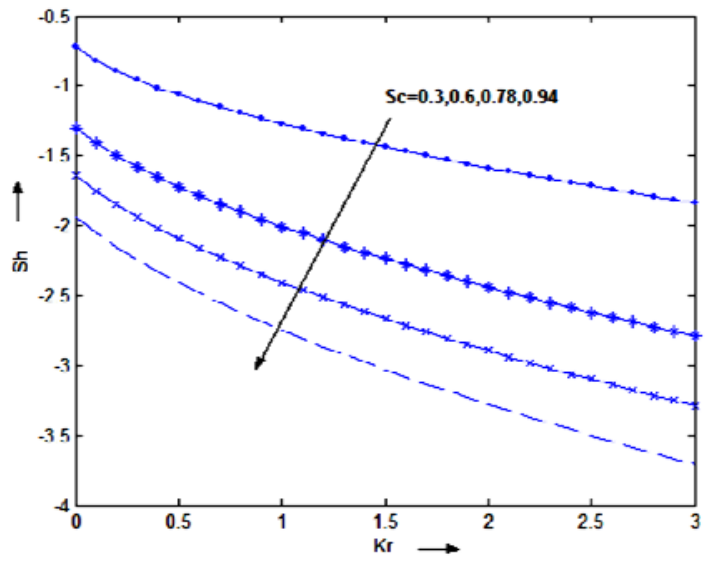

Fig.14. Effect of Sc on Sh against $K r$.

Table 1 represents a comparison of the numerical values of skin-friction $\tau$ obtained in the present case with those of Ravikumar et al. [21], Kim [20] for different values of non dimensional exponential index $n$. It is found that there is a good agreement. From Tab.2, it is of interest to note that the Nusselt number $(\mathrm{Nu})$ increases with increasing intensity of the radiation absorption $Q c$, however, an increase in the Schmidt number Sc, chemical reaction parameter $K r$, scalar constant $\varepsilon$, non-dimensional exponential index $n$, suction velocity parameter $A$, radiation parameter $R$ causes an decrease in the Nusselt number. In particular, the Nusselt number decreases with an increase in the Prandtl number Pr (this is in agreement with the result of Ref.[21])

Table 1. Comparison of the skin friction $(\tau)$ of present case with those of Ravikumar et al. [21], Kim [20] for different values of $n$.

\begin{tabular}{llll}
\hline$n$ & $\begin{array}{l}\text { Kim }[20] \\
(R m=0, K r=0,\end{array}$ & $\begin{array}{l}\text { Ravikumar } \text { et al. [21] } \\
(R m=0.1, K r=0,\end{array}$ & $\begin{array}{l}\text { Present work } \\
(R m=0.1, K r=0.1,\end{array}$ \\
& $R=0, G c=0, Q c=0)$ & $R=0, G c=0, Q c=0)$ & $\left.R=0.1, G c=0.1, Q_{c}=0.1\right)$ \\
\hline 1.0 & 3.9475 & 3.9508 & 3.9519 \\
0.1 & 2.8780 & 2.8671 & 2.8682 \\
0.01 & 2.8172 & 2.7653 & 2.7615 \\
\hline
\end{tabular}

Table 2. Effect of Sc, $K r, \varepsilon, n, A, \operatorname{Pr}, R$ and $Q c$ on the Nussult number $\mathrm{Nu}(t=1.0)$.

\begin{tabular}{lllllllll}
\hline $\mathrm{Sc}$ & $K r$ & $\varepsilon$ & $n$ & $A$ & $\mathrm{Pr}$ & $R$ & $Q c$ & $\mathrm{Nu}$ \\
\hline 0.6 & 0.5 & 0.2 & 1.0 & 0.5 & 0.71 & 0.1 & 0.01 & -1.6109 \\
0.78 & & & & & & & & -1.6124 \\
& 1.0 & & & & & & & -1.6135 \\
& & 0.3 & & & & & & -2.0232 \\
& & & 2.0 & & & & & -4.7603 \\
& & & & & 1.0 & & & -6.1524 \\
& & & & & & 0.2 & & -6.3146 \\
& & & & 0.7 & & & & -6.5464 \\
& & & & & & & 0.02 & -6.5314 \\
\hline
\end{tabular}

From Tab.3, it can be seen that the skin friction $\tau$ on the porous plate decreases with increasing values of magnetic field parameter $M$, Schimdt number Sc and radiation absorption parameter $Q c$, whereas it 
increases with an increase in all other parameters, in particular it increases with increasing the strength of suction velocity $(A)$ as well as the thermal buoyancy (Gr) (this is in agreement with the result of Ref.[21]).

Table 3. Effect of Gr, Gc, $M, U p, \mathrm{Sc}, \varepsilon, K r, n, A, \operatorname{Pr}, R, Q c$ and $K$ on skin friction $\tau(t=1.0)$.

\begin{tabular}{|c|c|c|c|c|c|c|c|c|c|c|c|c|c|}
\hline $\mathrm{Gr}$ & Gc & $M$ & $U p$ & $\mathrm{Sc}$ & $\varepsilon$ & $K r$ & $n$ & $A$ & $\operatorname{Pr}$ & $R$ & $Q c$ & $K$ & $\tau$ \\
\hline 1.0 & 1.0 & 2.0 & 0.5 & 0.6 & 0.2 & 0.5 & 1.0 & 0.5 & 0.71 & 0.1 & 0.01 & 100 & 4.0445 \\
\hline \multirow[t]{13}{*}{2.0} & & & & & & & & & & & & & 4.5498 \\
\hline & 2.0 & & & & & & & & & & & & 5.5323 \\
\hline & & 3.0 & & & & & & & & & & & 4.6761 \\
\hline & & & 0.6 & & & & & & & & & & 5.4559 \\
\hline & & & & 0.78 & & & & & & & & & 4.3238 \\
\hline & & & & & 0.3 & & & & & & & & 5.1244 \\
\hline & & & & & & 0.6 & & & & & & & 5.1426 \\
\hline & & & & & & & 2.0 & & & & & & 11.7657 \\
\hline & & & & & & & & 0.6 & & & & & 12.1926 \\
\hline & & & & & & & & & 1.0 & & & & 15.3086 \\
\hline & & & & & & & & & & & 0.02 & & 15.2970 \\
\hline & & & & & & & & & & 0.2 & & & 15.4582 \\
\hline & & & & & & & & & & & & 200 & 16.2547 \\
\hline
\end{tabular}

\section{Conclusion}

This paper studied the combined effects of radiation absorption and chemical reaction on a boundary layer flow of a Rivlin-Ericksen fluid with heat and mass transfer over a vertical moving porous plate. The thermal radiation, radiation absorption, transversely applied magnetic field and first order chemical reaction effects are taken into consideration. It is assumed that the temperature and the concentration at the wall as well as the suction velocity are exponentially varying with time. The governing equations of the problem were solved analytically by using the perturbation technique. A comprehensive set of graphical results for the velocity, temperature and concentration is presented and their dependence on physical parameters is discussed. Our results revealed that the velocity, temperature and Nusselt number increase and the skin friction decreases with an increase in radiation absorption. Furthermore, an increase in chemical reaction enhances the skin friction and reduces the Sherwood number and Nusselt number.

\section{Nomenclature}

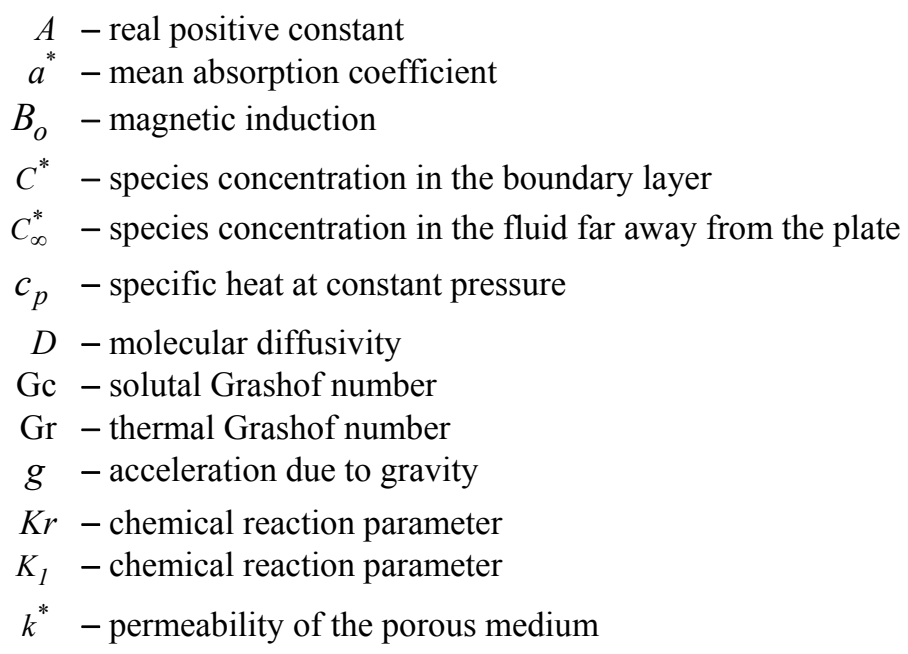


$M$ - magnetic field parameter

Pr - Prandtl number

$p^{*}$ - pressure

$R$ - radiation parameter

$R m$ - viscoelasticity parameter

Sc - Schmidt number

$T^{*}$ - temperature of the fluid in the boundary layer

$T_{\infty}^{*} \quad$ - temperature of the fluid far away from the plate

$T_{w}^{*}, C_{w}^{*}$ - wall temperature and concentration

$T_{\infty}^{*}, C_{\infty}^{*}$ - temperature and concentration

$t^{*}$ - dimensional time

$U_{p}^{*}$ - plate velocity

$U_{\infty}^{*} \quad$ - free steam velocity

$U_{0}, n^{*}$ - constants

$u^{*}, v^{*}$ - dimensional velocity components in $x^{*}, y^{*}$ directions

$Q c$ - radiation absorption parameter

$Q_{c}^{*}$ - coefficient of proportionality for the absorption of radiation

$q_{r}^{*} \quad$ - radiative heat flux

$x^{*}, y^{*}$ - dimensional distance along and perpendicular to the plate

$\rho$ - fluid density

$v$ - kinematic viscosity

$\alpha$ - thermal diffusivity

$\beta_{1}-$ kinematic visco-elasticity

$\beta_{T}, \beta_{C}-$ thermal and concentration expansion coefficients

$\varepsilon, \varepsilon A-$ small values less than unity

$\sigma$ - fluid electrical conductivity

$\sigma^{*}$ - Stefan-Boltzmann constant

\section{APPENDEX}

$$
\begin{aligned}
& m_{2}=\frac{\mathrm{Sc}+\sqrt{\mathrm{Sc}^{2}+4 K r \mathrm{Sc}}}{2}, \quad m_{4}=\frac{\mathrm{Sc}+\sqrt{\mathrm{Sc}^{2}+4(K r+n) \mathrm{Sc}}}{2}, \quad m_{6}=\frac{\operatorname{Pr}+\sqrt{\operatorname{Pr}^{2}+4 R \operatorname{Pr}}}{2}, \\
& m_{8}=\frac{\operatorname{Pr}+\sqrt{\operatorname{Pr}^{2}+4(R+n) \operatorname{Pr}}}{2}, \quad m_{10}=\frac{1+\sqrt{1+4 N}}{2}, \quad m_{12}=\frac{1+\sqrt{1+4(N+n)}}{2}, \\
& k_{1}=\frac{A \mathrm{Sc} m_{2}}{m_{2}^{2}-\mathrm{Sc} m_{2}-(K r+n) \mathrm{Sc}}, \quad k_{2}=1-k_{1}, \quad k_{3}=\frac{-Q c \operatorname{Pr}}{m_{2}^{2}-\operatorname{Pr} m_{2}-R \operatorname{Pr}}, \quad k_{4}=1-k_{3}, \\
& k_{5}=\frac{\operatorname{Pr} m_{6} k_{4} A}{m_{6}^{2}-\operatorname{Pr} m_{6}-(R+n) \operatorname{Pr}}, \quad k_{6}=\frac{-Q c \operatorname{Pr} k_{2}}{m_{4}^{2}-\operatorname{Pr} m_{4}-(R+n) \operatorname{Pr}}, \quad k_{7}=\frac{\operatorname{Pr}\left(m_{2} k_{3} A-k_{1} Q c\right)}{m_{2}^{2}-\operatorname{Pr} m_{2}-(R+n) \operatorname{Pr}},
\end{aligned}
$$




$$
\begin{aligned}
& k_{8}=1-k_{5}-k_{6}-k_{7}, \quad k_{9}=\frac{-\mathrm{Gr} k_{4}}{m_{6}^{2}-m_{6}-N}, \quad k_{10}=\frac{-\left(\mathrm{Gr} k_{3}+\mathrm{Gc}\right)}{m_{2}^{2}-m_{2}-N}, \\
& k_{11}=U_{p}-k_{9}-k_{10}-1, \quad k_{12}=\frac{k_{11} m_{10}^{3}}{m_{10}^{2}-m_{10}-N}, \quad k_{13}=\frac{k_{9} m_{6}^{3}}{m_{6}^{2}-m_{6}-N}, \\
& k_{14}=\frac{k_{10} m_{2}^{3}}{m_{2}^{2}-m_{2}-N}, \quad k_{15}=-k_{12}-k_{13}-k_{14}, \quad k_{16}=\frac{A k_{11} m_{10}}{m_{10}^{2}-m_{10}-(N+n)}, \\
& k_{17}=\frac{A k_{9} m_{6}-\mathrm{Gr} k_{5}}{m_{6}^{2}-m_{6}-(N+n)}, \quad k_{18}=\frac{A k_{10} m_{2}-\mathrm{Gr} k_{7}-\mathrm{Gc} k_{1}}{m_{2}^{2}-m_{2}-(N+n)}, \quad k_{19}=\frac{-\mathrm{Gr} k_{8}}{m_{8}^{2}-m_{8}-(N+n)}, \\
& k_{20}=\frac{-\mathrm{Gr} k_{6}-\mathrm{Gc} k_{2}}{m_{4}^{2}-m_{4}-(N+n)}, \quad k_{21}=-1-k_{16}-k_{17}-k_{18}-k_{19}-k_{20}, \quad k_{22}=\frac{k_{21} m_{12}^{3}+n k_{21} m_{12}^{2}}{m_{12}^{2}-m_{12}-(N+n)}, \\
& k_{23}=\frac{k_{16} m_{10}^{3}+n k_{16} m_{16}^{2}+A\left(k_{15}+k_{12}\right) m_{10}+A k_{11} m_{10}^{3}}{m_{10}^{2}-m_{10}-(N+n)}, \quad k_{24}=\frac{k_{17} m_{6}^{3}+n k_{17} m_{6}^{2}+A k_{13} m_{6}+A k_{9} m_{6}^{3}}{m_{6}^{2}-m_{6}-(N+n)}, \\
& k_{25}=\frac{k_{18} m_{2}^{3}+n k_{18} m_{2}^{2}+A k_{14} m_{2}+A k_{10} m_{2}^{3}}{m_{2}^{2}-m_{2}-(N+n)}, \quad k_{26}=\frac{k_{19} m_{8}^{3}+n k_{19} m_{8}^{2}}{m_{8}^{2}-m_{8}-(N+n)}, \\
& k_{27}=\frac{k_{20} m_{4}^{3}+n k_{20} m_{4}^{2}}{m_{4}^{2}-m_{4}-(N+n)}, \quad k_{28}=-k_{22}-k_{23}-k_{24}-k_{25}-k_{26}-k_{27} .
\end{aligned}
$$

\section{References}

[1] Scribben E., Baird D. and Wapperom P. (2006): The role of transient rheology in polymeric sintering. Rheologica Acta, vol.45, pp.825-839.

[2] Tichy J.A. (1996): Non-Newtonian lubrication with the convected Maxwell model. - ASMEJ. Tribol., vol.118, pp.344-348.

[3] Vlastos G.A. (1998): The visco-elastic behavior of blood and blood-like model fluids with emphasis on the superposition of steady and oscillatory shear. - Clinical. Hemorheology and Microcirculation, vol.19, pp.177179.

[4] Wouter Z., Hendriks M. and Hart M.T. (2005): A velocity-based approach to viscoelastic flow of rock. - Math. Geol, vol.37, pp.141-162.

[5] Nakayama A. and Koyama H. (1991): Buoyancy induced flow of a non-Newtonian fluids over a non-isothermal body of arbitrary shape in a fluid saturated porous medium. - Appl. Sci. Res, vol.48, pp.55--70.

[6] Mehta K.N. and Rao K.N. (1994): Buoyancy induced flow of a non-Newtonian fluids over a non isothermal horizontal plate embedded in a porous medium. - Int. J. Eng. Sci., vol.32, pp.521-525.

[7] Mehta K.N. and Rao K.N. (1994): Buoyancy induced flow of non-Newtonian fluids in a porous medium past a vertical plate with non-uniform surface heat flux. - Int. J. Eng. Sci., vol.32, pp.297-302. 
[8] De S., Kuipers J.A.M., Peters E.A.J.F. and Padding J.T. (2017): Viscoelastic flow simulations in random porous media. - Journal Of Non-Newtonian Fluid Mechanics, vol.248, pp.50-61.

[9] Bhukta D., Dash G.C. and Mishra S.R. (2014): Heat and mass transfer on MHD flow of a viscoelastic fluid through porous media over a shrinking sheet. - International Scholarly Research Notices, Article ID 572162, http://dx.doi.org/10.1155/2014/572162.

[10] Nayak M.K., Charan Dash G. and Prased Singh L. (2016): Heat and mass transfer effects on MHD visco elastic fluid over a stretching sheet through porous medium in presence of chemical reaction. - Propulsion and Power Research, vol.5, No.1, pp.70-80.

[11] Rivlin R.S. and Ericksen J.L. (1955): Stress -deformation relations for isotropic materials. - J. Rational Mechanics and Analysis, vol.4, pp.681-702.

[12] Srivastava R.K. and Singh K.K. (1988): Unsteady flow of a dusty elastico-viscous fluid through channels of different cross-sections in the presence of time-dependent pressure gradient. - Bull. Cal. Math. Soc., vol.80, pp.286.

[13] Garg A., Srivastava R.K. and Singh K.K. (1994): Drag on a sphere oscillating in conducting dusty Rivlin-Ericksen elastico-viscous liquid. - Proc. Nat. Acad. Sci. India, vol.64A, pp.355-363.

[14] Sharma R.C. and Kango S.K. (1999): Thermal convection in Rivlin-Ericksen elastico-viscous fluid in porous medium in hydromagnetics. - Czechoslovak Journal of Physics, vol.49, No.2, pp.197-203.

[15] Siddappa B. and Khapate B.S. (1976): Rivlin-Erickson fluid flow past stretching plate. - Rev Roum. Sci, Tech. Mech. Appl, vol.21, pp.497-505.

[16] Humera N., Ramana Murthy M.V., Reddy C.K., Rafiuddin M., Ramu A. and Rajender S. (2010): Hydromagneticsfree convective Rivlin-Ericksen flow through a porous medium with variable permeability. - Int. J. Comput. Appl. Math., vol.5, No.3, pp.267-75.

[17] Rana G.C. (2012): Thermal instability of compressible Rivlin-Efficksen rotating fluid permeated with suspended dust particles in porous medium. - Int. J. Appl. Math. Mech., vol.8, No.4, pp.97-110.

[18] Uwanta J. and Hussaini A. (2012): Effects of mass transfer on hydro magnetic free convective Rivlin-Ericksen flow through a porous medium with time dependent suction. - Int. J. Eng. Sci., vol.1, No.4, pp.21-30.

[19] Varshney N.K., Singh S. and Singh J. (2011): Effects of rotatory Rivlin-Ericksen fluid on MHD free convective and mass transfer flow through porous medium with constants heat and mass flux across moving plate. - IOSR J. Eng., vol.1, No.1, pp.10-7.

[20] Kim Youn J. (2000): Unsteady MHD convective heat transfer past a semi-infinite vertical porous moving plate with variable suction. - Int. J. Eng. Sci., vol.38, pp.833-845.

[21] Ravi Kumar V., Raju M.C. and Raju G.S.S. (2014): Combined effects of heat absorption and MHD on convective Rivlin-Erickese flow past a semi-infinite vertical plate with variable temperature and suction. - Ain Shams Engineering Journal, vol.5, No.3, pp.867-875.

[22] Sivaraj R. and Rushi Kumar B. (2013): Chemically reacting dusty viscoelastic fluid flow in an irregular channel with convective boundary. - Ain Shams Engineering Journal, vol.4, pp.93-101.

[23] Vicenti W.G. and Krugger G.H. (1965): Introduction to physical gas dynamics. - New York.

[24] Beard D.W. and Walters K. (1964): Elastico-viscous boundary-layer flows-I: two-dimensional flow near the stagnation point. - Proceedings of the Cambridge Philosophical Society, vol.60, pp.667-674.

Received: March 17, 2018

Revised: July 2, 2019 